\title{
OPEN Single cell RNA sequencing reveals ferritin as a key mediator of autoimmune pre-disposition in a mouse model of systemic lupus erythematosus
}

\author{
Subhi Talal Younes ${ }^{1}$, Kurt Showmaker ${ }^{2}$, Ashley C. Johnson ${ }^{2}$, Michael R. Garrett ${ }^{2}$ \& \\ Michael J. Ryan ${ }^{3 凶}$
}

Systemic lupus erythematosus (SLE) is a devastating autoimmune disorder characterized by failure of self-tolerance with resultant production of autoreactive antibodies. The etiology of this syndrome is complex, involving perturbations in immune cell signaling and development. The NZBWF1 mouse spontaneously develops a lupus-like syndrome and has been widely used as a model of SLE for over 60 years. The NZBWF1 model represents the F1 generation of a cross between New Zealand Black (NZB) and New Zealand White (NZW) mice. In order to better understand the factors that contribute to the development of autoimmunity, single cell RNA sequencing was conducted using the bone marrow from female NZBWF1 mice prior to the development of overt disease. The results were contrasted with single cell RNA sequencing results from the two parental strains. The expected findings of $B$ cell abundance and upregulation, and evidence of interferon signaling were validated in this model. In addition, several novel areas of inquiry were identified. Most notably, the data showed a marked upregulation of the ferritin light chain across all cell types in the NZBWF1 mice compared to parental controls. This data can serve as a gene expression atlas of all hematopoietic cells in the NZBWF1 bone marrow prior to the development of autoimmunity.

Systemic lupus erythematosus (SLE) is a multisystem autoimmune syndrome characterized in part by the production of a variety of auto-reactive antibodies ${ }^{1}$. The etiology of this complex syndrome is multi-factorial, involving both genetic susceptibility ${ }^{2}$ and complex environmental factors ${ }^{3}$. Consequently, the study of SLE has leveraged a variety of similarly multi-factorial model systems ${ }^{4}$ including mouse strains with polygenic susceptibility to autoimmunity, 5 . One such strain, the New Zealand Black-White F1 (NZBWF1), is produced by crossing a female New Zealand White (NZW) and male New Zealand Black (NZB). The resultant female F1 progeny exhibit an SLE-like phenotype characterized by production of anti-double stranded DNA antibodies and nephritis ${ }^{7}$.

Prior studies in the NZBWF1 model show multiple genetic susceptibility loci which collectively contribute to the phenotype $\mathrm{e}^{6,8}$. However, the precise mechanisms which contribute to the autoimmune predisposition in this strain are not clear. Importantly, the identification of genes that impart susceptibility to autoimmunity in NZBWF1 mice led to the discovery of orthologous genes linked to autoimmunity in humans ${ }^{9}$. Thus, the continued study of underlying genetic factors contributing to SLE in NZBWF1 mice can lead to a better understanding of human SLE. In order to advance this cause, single cell RNA sequencing was conducted using the bone marrow from 9 to 10 -week-old female NZBWF1 mice and the two parent strains, NZW and NZB. This age was selected because it precedes the development of overt autoimmunity. The purpose of this study was to define the genetic and cellular landscape of this model's hematopoietic system prior to the development of any disease phenotype. In so doing, several areas of novel inquiry were identified while simultaneously validating its use as a faithful small animal model of SLE.

\footnotetext{
${ }^{1}$ Department of Pediatrics, Boston Children's Hospital, Boston, MA, USA. ${ }^{2}$ Department of Pharmacology and Experimental Therapeutics, University of Mississippi Medical Center, Jackson, MS, USA. ${ }^{3}$ Department of Pharmacology, Physiology, and Neuroscience, University of South Carolina School of Medicine, 6439 Garners Ferry Road, Columbia, SC 29209, USA. ${ }^{\boxplus e m a i l: ~ M i c h a e l . R y a n 1 @ v a . g o v ~}$
} 


\section{Methods}

Animals. Seven-to-eight-week-old female New Zealand Black (NZB), New Zealand White (NZW), and New Zealand Black-White F1 (NZBWF1) mice $(\mathrm{n}=3)$ were purchased from The Jackson Laboratory, and were delivered at the same time. Animals were maintained on a 12-h day/night cycle and allowed to feed (standard chow) and drink ad libitum until they reached 9-10 weeks of age to conduct the study. Twenty-four hours prior to euthanasia, mice were placed in metabolic cages for collection of urine. At approximately 7:00 am, isoflurane was administered at a high dose until respirations ceased; cervical dislocation was then performed, and tissues were harvested. All studies were reviewed and approved by the University of Mississippi Medical Center Animal Care and Use Committee and comply with ARRIVE guidelines. All methods were carried out in accordance with relevant guidelines and regulations.

Bone marrow cell collection. The left and right femurs of the mice were disarticulated and dissected away from muscle. Each end of the bone was then clipped. Five milliliters of Hank's Balanced Salt Solution was flushed through each end of the bone using a 30-gauge needle. This solution was passed through a 70-micron filter to remove large pieces of debris. The cells were spun down at $200 \mathrm{rcf}$ for five minutes and re-suspended in $1 \mathrm{~mL}$ of phosphate-buffered saline (PBS) with $0.04 \%$ bovine serum albumin. Four milliliters of RBC lysis buffer from BD Biosciences (catalog number 555899) was added and the solution was incubated at room temperature for 5 min away from light. Cells were centrifuged again at $200 \mathrm{rcf}$ for $5 \mathrm{~min}$ and resuspended in $1 \mathrm{~mL}$ of PBS with $0.04 \%$ BSA. The centrifuged cells were washed three times according to the following protocol: centrifuge at $150 \mathrm{rcf}$ for $3 \mathrm{~min}$, remove the supernatant, resuspend the cells with a wide-bore pipette tip in $1 \mathrm{~mL}$ of PBS with $0.04 \%$ BSA. After the final wash and resuspension, cell concentration and viability was determined using a Bio-Rad TC20 automated cell counter.

Single cell RNA sequencing. Bone marrow cells were isolated using the $10 \times$ Genomics Chromium Single Cell controller according to the manufacturer's instructions using the $10 \times$ Chromium Single Cell 3' v3.1 Reagent Kit $(10 \times$ Genomics Product Code 1000268$)$ followed by cell lysis, and library preparation including barcoded RNA reverse transcription, cDNA amplification, addition of sample index, and Illumina P5/P7 adapters. The libraries were sequenced using an Illumina NextSeq 500 instrument. Reads from the NextSeq instrument were initially analyzed using the $10 \times$ Genomics Cell Ranger software v3.1. FastQ files were generated, samples demultiplexed, and gene counts obtained using the Cell Ranger mkfastq, count, and aggr commands. Resulting output tables were then imported into R statistical software for further analysis.

Data analysis. Data analysis was performed using $\mathrm{R}$ and a variety of Bioconductor packages ${ }^{10-30}$ following many of the conventions described by Amezquita and colleagues in the book Orchestrating Single Cell Analysis with Bioconductor ${ }^{31}$. The code associated with this analysis is available on GitHub (https://github.com/styounes/ SLE_scRNAseq).

HDF5 (.h5) files for each sample were loaded using the read10xCounts function of the DropletUtils package. Most of the following downstream analyses were performed using a combination of the scran and scater packages. Per cell quality control metrics included total detected unique molecular identifies (UMI), number of expressed genes, and the percentage of mitochondrial genes. Cells with less than 1000 total UMIs, less than 500 unique genes, or greater than $10 \%$ expression of mitochondrial genes were removed from the dataset. There was no difference in quality of cells among batches or across strains. These metrics exhibited a bimodal distribution (Supplementary Fig. 1A) with a clear inflection in between low- and high-quality cells, guiding the selection of thresholds. In order to ensure that highly metabolically active cells were not discarded by this approach, the total library size (i.e. total UMIs) was plotted against the percentage of mitochondrial genes for each cell (Supplementary Fig. 1B). Given the fact that there were no cells with large library sizes (presumably high-quality cells) with concomitant large proportion of mitochondrial genes, it was concluded that this approach did not inadvertently discard any metabolically active cell populations. Finally, the enrichment of gene subsets within discarded cells was checked and, observing no such enrichment, demonstrated that quality control was not discarding a distinct population of cells (Supplementary Fig. 1C). Doublets (droplets which contained two or more cells) were identified using a simulated doublet approach whereby artificial doublets are constructed from the dataset and cells which lie close to these artificial doublets in high-dimensional space are removed (guilt by association), as implemented in the scDblFinder function with the following non-default parameters: nfeatures $=750$, propRandom $=1$.

Gene expression was normalized by pooling counts from related cells and calculating a size factor for each pool; cells were then deconvolved into cell-based size factors for normalization of each cell's expression profile, using the functions quickCluster and computeSumfactors as implemented in the scran package. Genes with a high variance of expression after applying a correction factor for abundance were selected for use in several downstream analyses, most notably, dimensionality reduction. The top $20 \%$ of highly variable genes were selected.

Cell types were annotated using a curated set of marker genes garnered from the Cell Marker database (http:// bio-bigdata.hrbmu.edu.cn/CellMarker/) (Supplementary Table 1). Equipped with these gene sets, an assignment score for each permutation of cell and cell type was computed using the function AUCell with the following nondefault parameters: aucMaxRank $=750$. Diagnostic plots were inspected and score thresholds manually adjusted for optimal assignment in our dataset.

Differential gene expression among strains was determined by aggregating cell types across samples using the function aggregateAcrossCells implemented in the package edgeR. Samples with less than 10 cells for the given cell type were removed. Differential gene expression was computed by the function pseudoBulkDGE from the scran package. Briefly, this function loops across cell types and performs differential expression using 

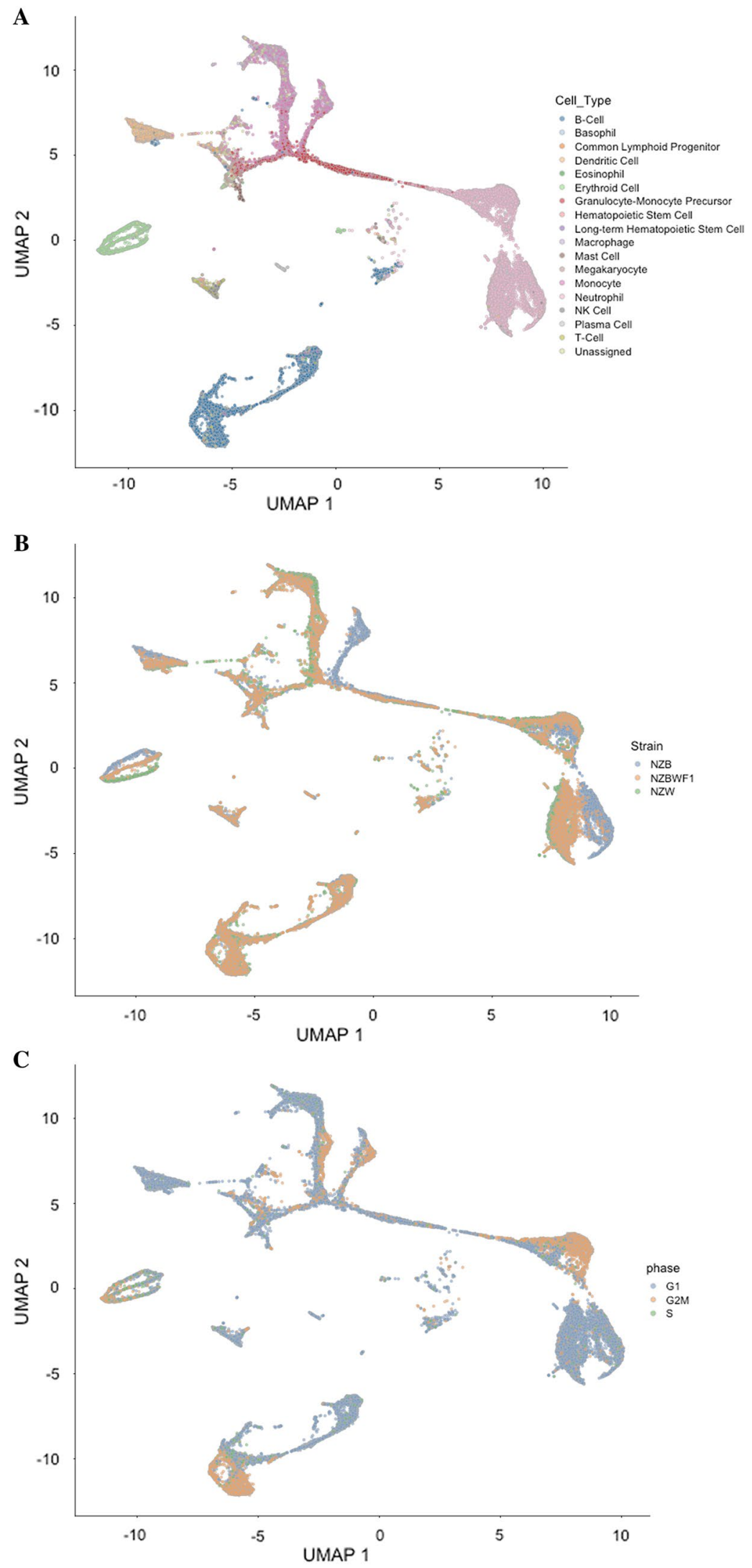

Figure 1. Dimensionality reduction of bone marrow cell gene expression. All plots are of the Uniform Manifold Approximation and Projection (UMAP). (A) Colored by assigned cell type. All three major hematopoietic lineages are present. (B) Colored by strain of origin for each cell. Note how the NZBWF1 cells often fall in between the NZW and NZB strains. (C) Colored by phase of the cell cycle. There is no significant difference in cell cycle phases between different strains. 


\begin{tabular}{|l|l|l|l|l|}
\hline Cell type & logFC & $\operatorname{logCPM}$ & F & P value \\
\hline B-cell & 0.98817355 & 17.6832439 & 17.9897336 & 0.00122998 \\
\hline Plasma cell & 1.27773038 & 14.113683 & 11.0312179 & 0.00703153 \\
\hline Mast cell & -0.4652928 & 12.7808536 & 3.31124615 & 0.0946657 \\
\hline T-cell & 0.68143326 & 14.6009994 & 3.2124122 & 0.10125933 \\
\hline Neutrophil & -0.3182438 & 18.3413162 & 2.56346925 & 0.13619568 \\
\hline Erythroid cell & -0.5468718 & 15.7460808 & 2.25148264 & 0.1622982 \\
\hline Granulocyte-monocyte precursor & -0.4410333 & 15.4648248 & 1.82167637 & 0.2048745 \\
\hline Hematopoietic stem cell & 0.35080046 & 12.5860995 & 1.75501409 & 0.2107284 \\
\hline Macrophage & 0.19599014 & 15.1284366 & 0.66806655 & 0.43017882 \\
\hline Monocyte & -0.1525407 & 16.8989742 & 0.34377706 & 0.56914649 \\
\hline Megakaryocyte & -0.1586588 & 14.3942908 & 0.24953066 & 0.62748041 \\
\hline Unassigned & 0.08894057 & 15.0939569 & 0.19374589 & 0.6678987 \\
\hline NK cell & 0.1427398 & 14.3660814 & 0.18558034 & 0.67513879 \\
\hline Dendritic cell & 0.05500362 & 16.0549614 & 0.07041496 & 0.79538582 \\
\hline
\end{tabular}

Table 1. Differential abundance of cell types across strains.

a quasi-likelihood method as implemented in the package edgeR. For the design matrix NZB-NZBWF1-NZW, the coefficients were: $-0.5,1,-0.5$. Similarly, differential abundance of cell populations was computed using quasi-likelihood method with a design matrix and coefficients as above. Phase of the cell cycle for each cell was inferred based on the expression of stereotypic cell cycle genes using the package cyclone.

Subclustering. B-cell subclusters were determined as follows. After appropriate subsetting of the global dataset, dimensionality reduction by principal component analysis was repeated. In order to minimize technical noise while maximizing identification of true biological differences, clustering was run using a subset of these principal components in keeping with the method described by Amezquita et al. ${ }^{30}$. The number of components was determined by first computing the number of clusters vs. the number of principal components used in clustering. Next, the number of principal components which yields no more than PC +1 clusters was selected, as it represents the inflection point between over- and under-clustering. A k-nearest-neighbor approach was applied to the dataset as implemented in the function buildSNNGraph in the igraph package (Supplementary Fig. 2A). Cluster stability was evaluated based on both cluster modularity and bootstrapping approaches (Supplementary Fig. 2B,C). One subcluster (label 1) was removed as this subcluster had a high library size and expressed both myeloid and lymphoid markers, suggesting it consisted mostly of doublets. Differential abundance and expression across B-cell subclusters was determined similar to that described above for other cell types.

\section{Results}

A high cell viability was obtained using the aforementioned isolation method, as NZB, NZW, and NZBWF1 mice had an average of 88,94 , and 94 percent viability, respectively. After initial quality control, 31,053 cells remained for downstream analysis. All three major hematopoietic lineages (immune, erythroid, and megakaryocytic) were represented across each individual strain (Fig. 1A,B). Interestingly, the gene expression of some NZBWF1 cell types-for example, erythroid and neutrophils-fell precisely in between the two parent strains (Fig. 1B), suggesting that the phenotype of the NZBWF1 strain is contributed to equally by both parent strains. By assessing the expression of stereotypic cell cycle genes, the phase of the cell cycle was assigned to each cell in our dataset (Fig. 1C). As expected, intermediate cell types (e.g. granulocyte-monocyte progenitors) exhibited progression through the cell cycle. There was no significant difference in the number of cells in each phase when compared amongst different strains (data not shown).

Next, the identification of any particular cell type exhibiting differential abundance in the NZBWF1 strain as compared to the NZB or NZW strain was examined. As shown in Table 1, B-cells and plasma cells from NZBWF1 mice exhibited an approximate onefold increase in abundance, supporting the key role of auto-antibody production in the SLE-like phenotype of this strain. Given this finding, a sub-cluster analysis of this B-cell population was conducted in order to discern whether any particular B-cell subtype is enriched in the NZBWF1 strain. Based on unsupervised clustering, there were 14 subtypes of B-cells present in the dataset (Fig. 2A). There was no significant difference in the abundance of any of these subtypes across strains (Fig. 2B). However, it is possible that the dataset may not be sufficiently powered to detect significant differences in these sub-populations. Similar to results for the entire dataset, NZBWF1 B-cells were not enriched in any given phase of the cell cycle when compared to the parent strains (data not shown).

In order to assess gene expression, a pseudo-bulk analysis used to identify genes which were differentially expressed in the NZBWF1 as compared to NZB and NZW strains within each cell type. Overall, very few genes were differentially expressed (Table 2 ). For example, in B-cells only 14 genes were significantly upregulated and only 3 were downregulated. Thus, the unique auto-immune phenotype of the NZBWF1 strain may be driven by relatively small alterations in gene expression as compared to its parent strains. Given the relatively small 

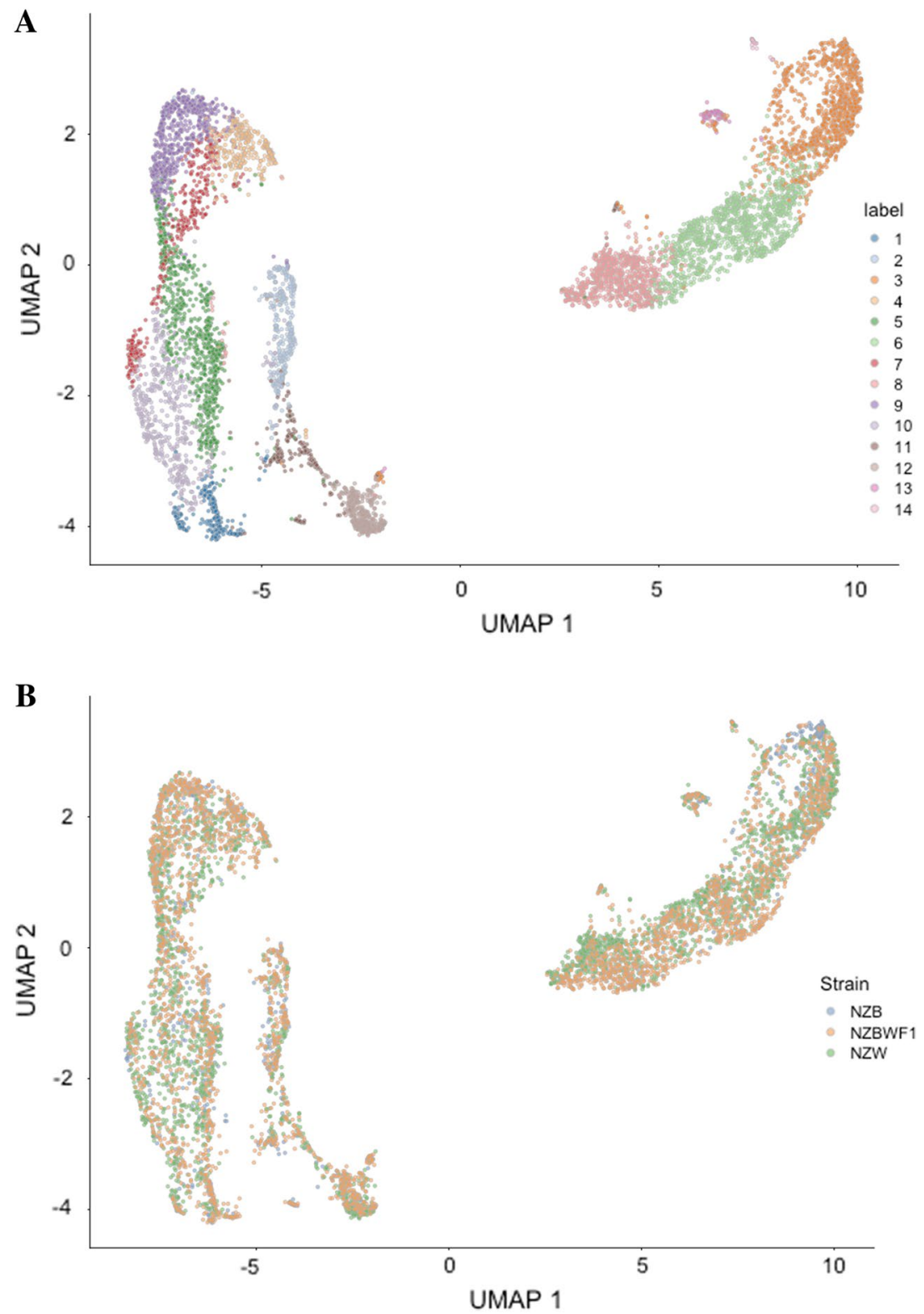

Figure 2. Dimensionality reduction of B-cells colored by cluster $(\mathbf{A})$ and strain $(\mathbf{B})$. There is no enrichment of any one cluster in the NZBWF1 strain as compared to the parent strains.

number of genes differentially expressed, gene ontology and reactome analyses failed to identify any significant enrichment for gene ontology terms or reactome pathways, respectively.

The specific genes which were differentially expressed were remarkably consistent across all cell types (Fig. 3, Table 3, and Supplementary Table 2). Among the most significant genes was Ftl1 and Ftl1-ps1, encoding ferritin light polypeptide 1 and ferritin light polypeptide 1 pseudogene 1 , respectively. Additional genes which exhibited consistent upregulation across multiple cell types were Ifitm2, Apobec3, and Ifi202b, all genes which are regulated by the interferon pathway. Ctse, the gene encoding Cathepsin E, a protein involved in MHC class II antigen presentation, was also consistently upregulated.

Several genes of cryptic functional significance were similarly upregulated including Tpm3-rs7, Htatip2, and Gm42031. Tpm3-rs7 (tropomyosin-related sequence 7) is a heretofore uncharacterized protein with putative actin filament binding. Gm42031 is an uncharacterized locus (postulated to be a lncRNA) whose downregulation in macrophages has been previously linked to neuroinflammation ${ }^{32}$. However, the dataset of this highly inflammation-prone strain shows that it is upregulated. Htatip2 is an oxidoreductase with a role in nuclear import signaling. There were no genes which exhibited consistent downregulation across all cell types.

Some genes were selectively altered in only specific cell types. For example, $L y 6 c 2$ expression was upregulated across myeloid cell types. Monocytes over-expressed several complement genes namely, C1qa and C1qb. Dendritic cells over-expressed $K l k 1$ (kallikrein). As expected, there were very few $\mathrm{T}$ cells in the bone marrow; 


\begin{tabular}{|l|l|l|l|l|}
\hline & Downregulated & Not Significant & Upregulated & NA \\
\hline B-cell & 3 & 9674 & 14 & 21,362 \\
\hline Dendritic cell & 2 & 6511 & 22 & 24,518 \\
\hline Erythroid cell & 2 & 8421 & 25 & 22,605 \\
\hline Granulocyte-monocyte precursor & 0 & 6900 & 14 & 24,139 \\
\hline Hematopoietic stem cell & 0 & 770 & 2 & 30,281 \\
\hline Macrophage & 0 & 5049 & 6 & 25,998 \\
\hline Mast cell & 0 & 927 & 2 & 30,124 \\
\hline Megakaryocyte & 0 & 4586 & 4 & 26,463 \\
\hline Monocyte & 16 & 8886 & 45 & 22,106 \\
\hline Neutrophil & 0 & 7930 & 24 & 23,099 \\
\hline NK cell & 1 & 2567 & 5 & 28,480 \\
\hline Plasma cell & 0 & 1410 & 5 & 29,638 \\
\hline T-cell & 0 & 2024 & 3 & 29,026 \\
\hline Unassigned & 1 & 5495 & 6 & 25,551 \\
\hline
\end{tabular}

Table 2. Number of differentially expressed genes across cell types.

thus, the lymphoid lineage was unable to be assessed as a whole. Regarding B-cells, $B c l 2 a 1 b$, an anti-apoptotic protein, was significantly downregulated in B-cells, suggesting that pro-survival signals may bypass immune checkpoints within the NZBWF1 mice. Plasma cells over-expressed Ighg $2 b$ and $I g h g 2 c$, further underscoring the central role of antibody production in the SLE-like phenotype.

\section{Discussion}

SLE is a multifactorial syndrome which involves a complex interaction of genetic susceptibility and environmental factors/exposures. By conducting single cell RNA sequencing on the bone marrow of the SLE-prone NZBWF1 mouse strain and contrasting it to its two parent strains, neither of which develop overt autoimmunity to the degree of their progeny, this study sought to unravel immune factors which contribute to this syndrome. Importantly, this analysis was conducted prior to the development of the disease phenotype, allowing for the identification of potential predisposing factors which contribute to later autoimmunity.

First, the results showed enrichment of B cells and plasma cells in the bone marrow of NZBWF1 mice. These $B$ cells expressed genes associated with activation and inflammation while the associated plasma cells exhibited upregulation of immunoglobulin $\mathrm{G}$ heavy chain. Taken together, these results underscore the central role of B cell activation and antibody production to the autoimmune phenotype of NZBWF $1^{33,34}$. A prior single cell RNA sequencing study of human peripheral blood in SLE $^{35}$ similarly identified alterations in B cells, noting enrichment of particular subpopulations of memory and activated B cells within the peripheral blood of SLE patients.

The interferon pathway is a well-known mediator of SLE in both mouse models ${ }^{36}$ and humans ${ }^{37-39}$. The finding of interferon activation further supports a key role for this pathway in the NZBWF1 and validates its use as a small animal model of SLE. The same study cited above ${ }^{35}$ also identified upregulation of the interferon pathway as of particular importance across a variety of cell types, including T-cells, monocytes, and dendritic cells in patients with SLE.

Of particular importance, our data showed marked upregulation of the ferritin light chain across all cell types in NZBWF1 mice relative to parental controls. Prior quantitative trait loci mapping identified a QTL associated with the SLE-like phenotype encompassing the Ftl1 locus on chromosome $7^{6,8}$. Playing a central role in iron uptake and storage ${ }^{40}$, the ferritin light chain is also a well-known acute phase reactant with important immune signaling actions ${ }^{41}$. Indeed, the ferritin light chain has been shown to be the primary circulating form of ferritin and mediates many of its immune functions ${ }^{42}$. Canonically, monocytes are the primary source of ferritin light chains in this context. Indeed, in the setting of autoimmunity, high levels of circulating ferritin are associated with the macrophage activation syndrome ${ }^{43,44}$. Thus, the marked upregulation across all cell types in this dataset is notable and represents a potentially novel avenue of scientific and therapeutic investigation. Whether ferritin upregulation is a cause of underlying NZBWF1 autoimmune predisposition remains to be elucidated.

Several genes of enigmatic function were also identified, suggesting potentially interesting areas of further study. Gm42031 was previously identified in a study of microglial activation. In that study, Wilson et al. identified its downregulation to be associated with neuro-inflammation ${ }^{32}$. Thus, the current finding of marked upregulation in the NZBWF1 strain in the context of inflammation is intriguing. Further characterization of Gm42031 and its functional impact on immune regulation may be a novel area of investigation.

To our knowledge, this is the first study to leverage single cell RNA sequencing in examining the bone marrow of the NZBWF1 mouse strain. Prior studies in this model have examined single cell transcriptomics in the kidney and lung of affected mice later in the disease course $\mathrm{e}^{45,46}$. There, the author's identified extensive immune cell infiltration and activation, consisting of nearly all major immune cell types. Thus, our study uniquely examines the developmental milieu of these immune cells at an early time point, prior to the development of overt autoimmunity.

Our study has several limitations. First, we examined only a single time point. It is possible earlier time points would have identified more proximate genetic contributors to autoimmune predisposition in the NZBWF1 strain. 

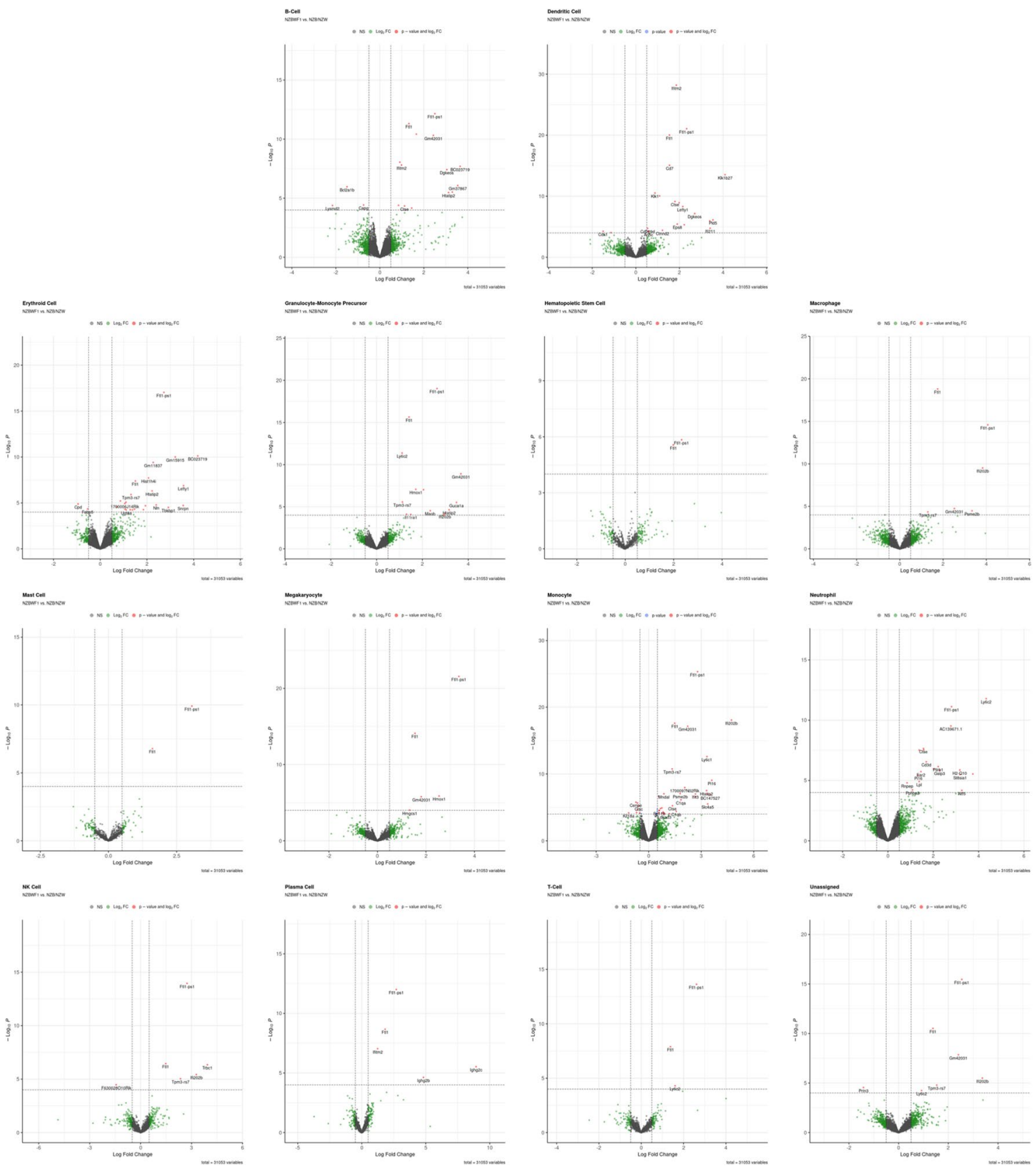

Figure 3. Volcano plots for each cell type.

Similarly, given this cross-sectional design, we cannot establish whether the observed gene expression changes cause autoimmunity. Future studies could examine longitudinal gene expression changes to more comprehensively chart the genetic and transcriptomic landscape of autoimmune development in the NZBWF1. Furthermore, targeted perturbations of the candidate pathways (e.g. ferritin light chain) could provide more insight into their causal role and establish therapeutic candidacy.

Taken together, the current data represent an atlas of hematopoietic phenotype early in the course of disease development of the NZBWF1 mouse. The data are not only consistent with the B cell activation and interferon signaling expected of SLE, but also identify several novel areas of interest, most notably, the ferritin light chain signaling axis. Data sets such as the one from this study may be useful for gaining a better understanding of this devastating disease leading to better therapeutic approaches. 


\begin{tabular}{|c|c|c|c|c|}
\hline Cell type & Gene & $\log \mathrm{FC}$ & P value & FDR \\
\hline \multirow{5}{*}{ B-cell } & Ftl1-ps1 & 2.50174162 & $7.07 \mathrm{E}-13$ & $6.86 \mathrm{E}-09$ \\
\hline & Ftl1 & 1.32126704 & $4.86 \mathrm{E}-12$ & $2.35 \mathrm{E}-08$ \\
\hline & Gm42031 & 2.4329193 & $4.81 \mathrm{E}-11$ & $1.17 \mathrm{E}-07$ \\
\hline & Tpm3-rs7 & 1.65835559 & $3.80 \mathrm{E}-11$ & $1.17 \mathrm{E}-07$ \\
\hline & Apobec3 & 0.91044418 & \begin{tabular}{|l|l|}
$8.94 \mathrm{E}-09$ \\
\end{tabular} & $1.73 \mathrm{E}-05$ \\
\hline \multirow{5}{*}{ Dendritic cell } & Ifitm2 & 1.85651713 & $6.29 \mathrm{E}-29$ & $4.11 \mathrm{E}-25$ \\
\hline & Ftl1-ps1 & 2.33121597 & $8.69 \mathrm{E}-22$ & $2.84 \mathrm{E}-18$ \\
\hline & Ftl1 & 1.53974644 & $9.65 \mathrm{E}-21$ & $2.10 \mathrm{E}-17$ \\
\hline & $\mathrm{Cd} 7$ & 1.54503052 & $8.59 \mathrm{E}-16$ & $1.40 \mathrm{E}-12$ \\
\hline & Klk1b27 & 4.08987062 & $2.93 \mathrm{E}-14$ & $3.83 \mathrm{E}-11$ \\
\hline \multirow{5}{*}{ Erythroid cell } & Ftl1-ps1 & 2.72595882 & $9.11 \mathrm{E}-18$ & 7.69E-14 \\
\hline & Gm15915 & 3.21054274 & $9.91 \mathrm{E}-11$ & $2.79 \mathrm{E}-07$ \\
\hline & BC023719 & 4.17275982 & $7.45 \mathrm{E}-11$ & $2.79 \mathrm{E}-07$ \\
\hline & Gm11837 & 2.26635837 & $3.85 \mathrm{E}-10$ & $8.14 \mathrm{E}-07$ \\
\hline & Histlh4i & 2.06363105 & $1.95 \mathrm{E}-08$ & $3.29 \mathrm{E}-05$ \\
\hline \multirow{5}{*}{ Granulocyte-monocyte precursor } & Ftl1-ps1 & 2.63673211 & $9.33 \mathrm{E}-20$ & $6.45 \mathrm{E}-16$ \\
\hline & Ftll & 1.41551313 & $2.22 \mathrm{E}-16$ & $7.67 \mathrm{E}-13$ \\
\hline & Ly6c2 & 1.11070042 & $4.23 \mathrm{E}-12$ & $9.74 \mathrm{E}-09$ \\
\hline & Gm42031 & 3.68043299 & $1.26 \mathrm{E}-09$ & $2.17 \mathrm{E}-06$ \\
\hline & Hmox1 & 1.70928675 & $8.60 \mathrm{E}-08$ & 0.00011017 \\
\hline \multirow{2}{*}{ Hematopoietic stem cell } & Ftl1 & 1.98368353 & $2.70 \mathrm{E}-06$ & 0.00104082 \\
\hline & Ftl1-ps1 & 2.32695056 & $1.45 \mathrm{E}-06$ & 0.00104082 \\
\hline \multirow{5}{*}{ Macrophage } & Ftll & 1.75021657 & $1.53 \mathrm{E}-19$ & $7.74 \mathrm{E}-16$ \\
\hline & Ftl1-ps1 & 4.06719941 & $2.59 \mathrm{E}-15$ & $6.56 \mathrm{E}-12$ \\
\hline & Ifi202b & 3.83046466 & $3.01 \mathrm{E}-10$ & $5.07 \mathrm{E}-07$ \\
\hline & Gm42031 & 2.52142643 & $1.81 \mathrm{E}-05$ & 0.02281615 \\
\hline & Psme2b & 3.3391237 & $3.31 \mathrm{E}-05$ & 0.03350592 \\
\hline \multirow{2}{*}{ Mast cell } & Ft11-ps1 & 3.05990462 & $1.22 \mathrm{E}-10$ & $1.13 \mathrm{E}-07$ \\
\hline & Ftl1 & 1.6133699 & \begin{tabular}{|l|}
$1.65 \mathrm{E}-07$ \\
\end{tabular} & 7.66E-05 \\
\hline \multirow{4}{*}{ Megakaryocyte } & Ftl1-ps1 & 3.354989 & $2.69 \mathrm{E}-22$ & $1.23 \mathrm{E}-18$ \\
\hline & Ftl1 & 1.54781558 & \begin{tabular}{|l}
$7.85 \mathrm{E}-15$ \\
\end{tabular} & $1.80 \mathrm{E}-11$ \\
\hline & Hmox1 & 2.53794703 & $1.33 \mathrm{E}-06$ & 0.00189498 \\
\hline & Gm42031 & 1.8046175 & $1.65 \mathrm{E}-06$ & 0.00189498 \\
\hline \multirow{5}{*}{ Monocyte } & Ftl1-ps1 & 2.78859068 & $4.67 \mathrm{E}-26$ & $4.18 \mathrm{E}-22$ \\
\hline & Ifi202b & 4.72648063 & $8.41 \mathrm{E}-19$ & $3.76 \mathrm{E}-15$ \\
\hline & Ftll & 1.48626521 & \begin{tabular}{|l|}
$2.47 \mathrm{E}-18$ \\
\end{tabular} & 7.37E-15 \\
\hline & Gm42031 & 2.22773198 & \begin{tabular}{|l}
$7.12 \mathrm{E}-18$ \\
\end{tabular} & $1.59 \mathrm{E}-14$ \\
\hline & Ly6c1 & 3.32351574 & $2.51 \mathrm{E}-13$ & $4.49 \mathrm{E}-10$ \\
\hline \multirow{5}{*}{ Neutrophil } & Ly6c2 & 4.33353168 & $1.60 \mathrm{E}-12$ & $1.27 \mathrm{E}-08$ \\
\hline & Ftl1-ps1 & 2.80701994 & $7.69 \mathrm{E}-12$ & $3.06 \mathrm{E}-08$ \\
\hline & AC139671.1 & 2.77693183 & $3.03 \mathrm{E}-10$ & $8.02 \mathrm{E}-07$ \\
\hline & Ctse & 1.56917145 & \begin{tabular}{|l|}
$2.23 \mathrm{E}-08$ \\
\end{tabular} & 3.99E-05 \\
\hline & Ftl1 & 1.5759339 & \begin{tabular}{|l|}
$2.90 \mathrm{E}-08$ \\
\end{tabular} & 3.99E-05 \\
\hline \multirow{5}{*}{ NK cell } & Ftl1-ps1 & 2.73386052 & $1.11 \mathrm{E}-14$ & $2.85 \mathrm{E}-11$ \\
\hline & Trbcl & 3.92664566 & $4.52 \mathrm{E}-07$ & 0.00038791 \\
\hline & Ftl1 & $\mid 1.47947574$ & $3.58 \mathrm{E}-07$ & 0.00038791 \\
\hline & Ifi202b & 3.2806935 & 3.72E-06 & 0.00239273 \\
\hline & \begin{tabular}{|l|} 
Tpm3-rs7 \\
\end{tabular} & 2.34843827 & \begin{tabular}{|l|l|}
$9.22 \mathrm{E}-06$ \\
\end{tabular} & \begin{tabular}{|l|}
0.00474277 \\
\end{tabular} \\
\hline \multirow{5}{*}{ Plasma cell } & Ftl1-ps1 & 2.6977062 & \begin{tabular}{|l}
$9.79 \mathrm{E}-13$ \\
\end{tabular} & \begin{tabular}{|l|}
$1.39 \mathrm{E}-09$ \\
\end{tabular} \\
\hline & Ftll & 1.82543793 & $2.12 \mathrm{E}-09$ & $1.50 \mathrm{E}-06$ \\
\hline & Ifitm2 & 1.24298817 & $9.23 \mathrm{E}-08$ & $4.35 \mathrm{E}-05$ \\
\hline & Ighg2c & 8.93073673 & $2.95 \mathrm{E}-06$ & \begin{tabular}{|l|}
0.00104291 \\
\end{tabular} \\
\hline & Ighg2b & 4.82008471 & $2.36 \mathrm{E}-05$ & 0.006666555 \\
\hline \multirow{3}{*}{ T-cell } & Ftl1-ps1 & 2.60830889 & 2.32E-14 & \begin{tabular}{|l|}
$4.70 \mathrm{E}-11$ \\
\end{tabular} \\
\hline & Ftl1 & 1.37918593 & \begin{tabular}{|l|}
$1.27 \mathrm{E}-08$ \\
\end{tabular} & $1.29 \mathrm{E}-05$ \\
\hline & Ly6c2 & 1.59713348 & $4.96 \mathrm{E}-05$ & 0.03351994 \\
\hline
\end{tabular}

Table 3. Top differentially expressed genes for each cell type as depicted in Fig. 3. The top 5 most significant genes are listed. For cell types with less than 5 significant genes, only those genes of statistical significance are shown. 


\section{Data availability}

Raw data has been deposited in the NCBI-GEO under accession number GSE174728 (https://www.ncbi.nlm. nih.gov/geo/query/acc.cgi?acc=GSE174728). Code for data analysis is available in GitHub (https://github.com/ styounes/SLE_scRNAseq).

Received: 20 May 2021; Accepted: 13 October 2021

Published online: 20 December 2021

\section{References}

1. Tsokos, G. C. Mechanisms of disease: Systemic lupus erythematosus. N. Engl. J. Med. https://doi.org/10.1056/NEJMra1100359 (2011).

2. Moser, K. L., Kelly, J. A., Lessard, C. J. \& Harley, J. B. Recent insights into the genetic basis of systemic lupus erythematosus. Genes Immun. 10, 373-379 (2009).

3. Cooper, G. S. et al. Hormonal, environmental, and infectious risk factors for developing systemic lupus erythematosus. Arthritis Rheum. https://doi.org/10.1002/1529-0131(199810)41:10\%3c1714::AID-ART3\%3e3.0.CO;2-U (1998).

4. Richard, M. L. \& Gilkeson, G. Mouse models of lupus: What they tell us and what they don't. Lupus Sci. Med. https://doi.org/10. 1136/lupus-2016-000199 (2018).

5. Andrews, B. S. et al. Spontaneous murine lupus-like syndromes: Clinical and immunopathological manifestations in several strains*. J. Exp. Med. https://doi.org/10.1084/jem.148.5.1198 (1978).

6. Morel, L., Rudofsky, U. H., Longmate, J. A., Schiffenbauer, J. \& Wakeland, E. K. Polygenic control of susceptibility to murine systemic lupus erythematosus. Immunity 1, 219-229 (1994).

7. Dixon, F. J. et al. Etiology and pathogenesis of a spontaneous lupus-like syndrome in mice. Arthritis Rheum. https://doi.org/10. 1002/art.1780210909 (1978)

8. Mohan, C., Yu, Y., Morel, L., Yang, P. \& Wakeland, E. K. Genetic dissection of Sle pathogenesis: Sle3 on murine chromosome 7 impacts T cell activation, differentiation, and cell death. J. Immunol. 162, 6492-6502 (1999).

9. Morel, L. Genetics of SLE: Evidence from mouse models. Nat. Rev. Rheumatol. 6, 348-357 (2010).

10. Wickham, H. et al. Welcome to the Tidyverse. J. Open Source Softw. https://doi.org/10.21105/joss.01686 (2019).

11. Amezquita, R. A. et al. Orchestrating single-cell analysis with bioconductor. Nat. Methods 17, 137-145 (2020).

12. Lun, A. T. L. et al. EmptyDrops: Distinguishing cells from empty droplets in droplet-based single-cell RNA sequencing data. Genome Biol. 20, 63 (2019).

13. Griffiths, J. A., Richard, A. C., Bach, K., Lun, A. T. L. \& Marioni, J. C. Detection and removal of barcode swapping in single-cell RNA-seq data. Nat. Commun. 9, 2667 (2018).

14. McCarthy, D. J., Campbell, K. R., Lun, A. T. L. \& Wills, Q. F. Scater: Pre-processing, quality control, normalization and visualization of single-cell RNA-seq data in R. Bioinformatics https://doi.org/10.1093/bioinformatics/btw777 (2017).

15. Morgan, M. \& Carlson, M. AnnotationHub: Client to access AnnotationHub resources. R package version 2.22.0. (2020).

16. Lun, A. T. L., McCarthy, D. J. \& Marioni, J. C. A step-by-step workflow for low-level analysis of single-cell RNA-seq data with bioconductor. F1000 Res. 5, 2122 (2016).

17. Robinson, M. D., McCarthy, D. J. \& Smyth, G. K. edgeR: A bioconductor package for differential expression analysis of digital gene expression data. Bioinformatics 26, 139-140 (2010).

18. McCarthy, D. J., Chen, Y. \& Smyth, G. K. Differential expression analysis of multifactor RNA-Seq experiments with respect to biological variation. Nucleic Acids Res. 40, 4288-4297 (2012).

19. Blighe, K. \& Lun, A. PCAtools: Everything Principal Components Analysis. R package version 2.2.0. (2020).

20. Morgan, M., Obenchain, V., Lang, M., Thompson, R. \& Turaga, N. BiocParallel: Bioconductor facilities for parallel evaluation. $R$ package version 1.24.1. (2020).

21. Kolde, R. pheatmap: Pretty Heatmaps. R package version 1.0.12. (2019).

22. Aran, D. et al. Reference-based analysis of lung single-cell sequencing reveals a transitional profibrotic macrophage. Nat. Immunol. 20, 163-172 (2019).

23. Trapnell, C. et al. The dynamics and regulators of cell fate decisions are revealed by pseudotemporal ordering of single cells. Nat. Biotechnol. https://doi.org/10.1038/nbt.2859 (2014).

24. Cao, J. et al. The single-cell transcriptional landscape of mammalian organogenesis. Nature 566, 496-502 (2019).

25. Qiu, X. et al. Reversed graph embedding resolves complex single-cell trajectories. Nat. Methods 14, 979-982 (2017).

26. Germain, P. scDblFinder. R package version 1.4.0. (2020).

27. Alexa, A. \& Rahnenfuhrer, J. topGO: Enrichment analysis for gene ontology. R package version 2.42.0. (2020).

28. Blighe, K., Rana, S., Turkes, E., Ostendorf, B. \& Lews, M. EnhancedVolcano: Publication-ready volcano plots with enhanced colouring and labeling. $R$ package version 1.8.0.

29. Ritchie, M. E. et al. Limma powers differential expression analyses for RNA-sequencing and microarray studies. Nucleic Acids Res. https://doi.org/10.1093/nar/gkv007 (2015).

30. R Core Team. R: A Language and Environment for Statistical Computing. (R Core Team, 2021).

31. Amezquita, R., Lun, A., Hicks, S. \& Gottardo, R. Orchestrating single-cell analysis with bioconductor. Bioconductor 14, e1006378 (2020).

32. Wilson, K., Miranda, K., Kaul, M., Nagarkatti, P. \& Nagarkatti, M. Single cell profiling illustrates down-regulation of GM42031 in macrophages and microglia as a potential mechanism of neuroinflammation in transgenic GFAP-gp120 mice. J. Immunol. 204, 225 (2020).

33. Haas, K. M. et al. Protective and pathogenic roles for b cells during systemic autoimmunity in NZB/W F 1 mice. J. Immunol. https:// doi.org/10.4049/jimmunol.0902391 (2010).

34. Taylor, E. B., Barati, M. T., Powell, D. W., Turbeville, H. R. \& Ryan, M. J. Plasma cell depletion attenuates hypertension in an experimental model of autoimmune disease. Hypertension https://doi.org/10.1161/HYPERTENSIONAHA.117.10473 (2018),

35. Nehar-Belaid, D. et al. Mapping systemic lupus erythematosus heterogeneity at the single-cell level. Nat. Immunol. 21, 1094-1106 (2020).

36. Liu, Z. et al. IFN- $\alpha$ confers resistance of systemic lupus erythematosus nephritis to therapy in NZB/W F1 mice. J. Immunol. https:// doi.org/10.4049/jimmunol.1004142 (2011).

37. Baechler, E. C. et al. Interferon-inducible gene expression signature in peripheral blood cells of patients with severe lupus. Proc. Natl. Acad. Sci. USA. https://doi.org/10.1073/pnas.0337679100 (2003).

38. Berry, M. P. R. et al. An interferon-inducible neutrophil-driven blood transcriptional signature in human tuberculosis. Nature https://doi.org/10.1038/nature09247 (2010).

39. Bennett, L. et al. Interferon and granulopoiesis signatures in systemic lupus erythematosus blood. J. Exp. Med. https://doi.org/10. 1084/jem.20021553 (2003).

40. Arosio, P. \& Levi, S. Cytosolic and mitochondrial ferritins in the regulation of cellular iron homeostasis and oxidative damage. Biochim. Biophys. Acta Gen. Subj. https://doi.org/10.1016/j.bbagen.2010.02.005 (2010). 
41. Wang, W., Knovich, M. A., Coffman, L. G., Torti, F. M. \& Torti, S. V. Serum ferritin: Past, present and future. Biochim. Biophys. Acta Gen. Subj. https://doi.org/10.1016/j.bbagen.2010.03.011 (2010).

42. Cohen, L. A. et al. Serum ferritin is derived primarily from macrophages through a nonclassical secretory pathway. Blood https:// doi.org/10.1182/blood-2009-11-253815 (2010).

43. Allen, C. E., Yu, X., Kozinetz, C. A. \& McClain, K. L. Highly elevated ferritin levels and the diagnosis of hemophagocytic lymphohistiocytosis. Pediatr. Blood Cancer https://doi.org/10.1002/pbc.21423 (2008).

44. Wong, K. The acute lupus hemophagocytic syndrome. Ann. Intern. Med. 114, 387 (1991).

45. Bates, M. A. et al. Mapping of dynamic transcriptome changes associated with silica-triggered autoimmune pathogenesis in the lupus-prone NZBWF1 mouse. Front. Immunol. 10, 632 (2019).

46. Der, E. et al. Single cell RNA sequencing to dissect the molecular heterogeneity in lupus nephritis. JCI Insight 2, 9 (2017).

\section{Acknowledgements}

This work was supported by National Institutes of Health Grants R01-HL136684 (to M. J. Ryan), P01-HL-051971 and U54-GM-115428 (to the University of Mississippi Medical Center Department of Physiology and Biophysics). The work performed through the UMMC Molecular and Genomics Facility is supported, in part, by funds from the NIGMS, including Mississippi INBRE (P20GM103476), Obesity, Cardiorenal and Metabolic DiseasesCOBRE (P20GM104357).

\section{Author contributions}

M.J.R. and S.T.Y. conceived of the study. M.J.R., M.R.G., and S.T.Y. designed the study. S.T.Y. and A.C.J. collected data and performed the experiments. S.T.Y., K.S., and M.R.G. analyzed the data. S.T.Y. wrote the first draft of the manuscript. All authors contributed to the interpretation of the results, revised the manuscript, and have read and approved the final manuscript for submission.

\section{Competing interests}

The authors declare no competing interests.

\section{Additional information}

Supplementary Information The online version contains supplementary material available at https://doi.org/ 10.1038/s41598-021-03649-2.

Correspondence and requests for materials should be addressed to M.J.R.

Reprints and permissions information is available at www.nature.com/reprints.

Publisher's note Springer Nature remains neutral with regard to jurisdictional claims in published maps and institutional affiliations.

(c) (i) Open Access This article is licensed under a Creative Commons Attribution 4.0 International License, which permits use, sharing, adaptation, distribution and reproduction in any medium or format, as long as you give appropriate credit to the original author(s) and the source, provide a link to the Creative Commons licence, and indicate if changes were made. The images or other third party material in this article are included in the article's Creative Commons licence, unless indicated otherwise in a credit line to the material. If material is not included in the article's Creative Commons licence and your intended use is not permitted by statutory regulation or exceeds the permitted use, you will need to obtain permission directly from the copyright holder. To view a copy of this licence, visit http://creativecommons.org/licenses/by/4.0/.

(C) The Author(s) 2021 